\title{
ROSAT observations of the soft X-ray background and of the cluster soft excess emission in the Hercules supercluster
}

\author{
M. Bonamente ${ }^{1,2}$, R. Lieu ${ }^{2}$, and J. Kaastra ${ }^{3}$ \\ 1 NASA National Space Science Technology Center, Huntsville, AL, USA \\ e-mail: bonamem@uah.edu \\ 2 Department of Physics, University of Alabama, Huntsville, AL, USA \\ e-mail: lieur@cspar.uah.edu \\ 3 SRON, Utrecht, The Netherlands \\ e-mail: J.S.Kaastra@sron.nl
}

Received 1 April 2005 / Accepted 28 July 2005

\begin{abstract}
Recent observations with the XMM-Newton satellite confirmed the existence of the soft excess phenomenon in galaxy clusters, earlier discovered in several EUVE, ROSAT and BeppoSAX observations. Among the clusters for which XMM has reported detection of soft excess emission are MKW03s and A2052, two clusters in the Hercules concentration.

The Hercules supercluster lies along the southern extension of the North Polar Spur, a region of bright soft X-ray emission clearly visible in ROSAT All-Sky Survey images. We analyze 11 pointed ROSAT PSPC observations toward 3 clusters in the Hercules concentration, MKW03s, A2052 and A2063, and 8 neighboring fields in order to investigate the soft X-ray emission in that region of the sky. We find that the soft X-ray emission varies by a factor of few on scales of few degrees, rendering the background subtraction a complex task. If the North Polar Spur emission is of local origin, we find that only A2052 and A2063 have evidence of cluster soft excess emission, and that the OVII emission lines detected in XMM observations of A2052 and MKW03s are not associated with the cluster. If part or all of the North Polar Spur soft X-ray enhancement is of extragalactic nature, the three clusters feature strong soft excess emission, and the OVII emission lines observed by XMM are genuinely associated with the clusters.

We interpret the soft excess emission with the presence of warm gas, either intermixed with the hot intra-cluster medium or in filamentary structures located around the clusters, and estimate that the warm gas is approximately as massive as the hot intra-cluster medium.
\end{abstract}

Key words. galaxies: clusters: individual: MKW03s, A2052, A2063 - X-rays: galaxies: clusters

\section{Introduction}

Excess of soft X-ray emission from galaxy clusters, above the contribution from the hot $\left(T \sim 10^{7}-10^{8} \mathrm{~K}\right)$ intra-cluster medium (ICM), is commonplace. Analyses of large samples of galaxy clusters (Bonamente et al. 2002; Kaastra et al. 2003a,b; Nevalainen et al. 2003) indicate that the phenomenon is detected in about $30-50 \%$ of the clusters. Among the clusters that have reported soft excess emission to date are Coma (Lieu et al. 1996a; Bonamente et al. 2003), Virgo (Lieu et al. 1996b), A1795 (Mittaz et al. 1999; Bonamente et al. 2001aa), A2199 (Lieu et al. 1999; Bonamente et al. 2001aa; Kaastra et al. 2001), Sérsic 159-03 (Bonamente et al. 2001bb; Kaastra et al. 2003a,b), a few clusters in the Shapley concentration (Bonamente et al. 2001c), two clusters in the Hercules concentration, MKW03s and A2052 (Kaastra et al. 2003a,b), A3112 (Nevalainen et al. 2003) and a few more clusters detected at lower significance (Bonamente et al. 2002).
Detection of soft excess radiation from clusters relies on a correct background subtraction. Inhomogenieties in the diffuse soft X-ray background on a scale of few degrees are common across the whole sky. In addition, variations in the $1 / 4 \mathrm{keV}$ band are not necessarily correlated with variations in the neighboring $3 / 4 \mathrm{keV}$ band, indicating that the soft X-ray background originates from a multi-phase plasma (Snowden et al. 2000). The background subtraction task is particularly complex in the case of the Hercules supercluster due to the presence of an extended emission feature along the line of sight, the North Polar Spur.

In this paper we analyze the ROSAT PSPC data of 3 clusters in the Hercules supercluster, MKW03s, A2052 and A2063 and of 8 neighboring background fields. Our PSPC data covers larger areas than the available XMM-Newton observations. With these data, we quantify the level of soft X-ray background fluctuations near the Hercules supercluster, and obtain several in situ background measurements in order to investigate the 
effect of background subtraction on the determination of soft excess fluxes. The ROSAT data presented in this paper reveals important new information concerning the reality and the nature of the excess emission in the Hercules supercluster.

The paper is structured in the following way. In Sect. 2 we provide a summary of the earlier XMM-Newton results for the clusters MKW03s and A2052, in Sect. 3 we present the PSPC data and in Sect. 4 we present our results on the soft X-ray emission from the North Polar Spur. In Sect. 5 we discuss the presence of soft excess emission from the three clusters in the Hercules concentration, in Sect. 6 we provide an interpretation of the excess emission and Sect. 7 contains our conclusions. In this paper we use a $\Omega_{b}=0.04, \Omega_{\mathrm{m}}=0.3$ and $\Omega_{\Lambda}=0.7$ cosmology, and a Hubble constant of $H_{0}=72 \mathrm{~km} \mathrm{~s}^{-1} \mathrm{Mpc}^{-1}$. The redshifts of the 3 clusters are $z=0.045$ (MKW03s) and $z=0.035$ (A2052 and A2063).

\section{XMM-Newton results on the soft excess in the Hercules supercluster}

Kaastra et al. (2003a,b) reported the presence of strong soft excess emission in the XMM-Newton data of two clusters in the Hercules concentration, MKW03s and A2052. The excess emission at large radii ( $\geq 4$ arcmin) features apparent OVII emission lines that Kaastra et al. (2003a) attributes to the soft excess component. For the purpose of background subtraction Kaastra et al. (2003a,b) used the standard background datasets of Lumb et al. (2002), composed of several observations at random orientations of low Galactic absorption, with the addition of a $35 \%$ statistical error in an effort to account for possible background variations.

A study of the same XMM-Newton observations by Mittaz et al. (2005) featured a local background instead, which was estimated from peripheral regions of the cluster observation. This analysis shows that, while the presence of the excess emission is confirmed, the detection of OVII emission lines is not. In fact, the Galactic soft X-ray background also features similar $z=0$ emission lines that an incorrect background subtraction will attribute to the soft excess emitter.

Given that the reality of the excess emission and of the associated OVII emission lines depends on a correct background subtraction, we address this issue in more detail using PSPC observations of these two clusters, of A2063 (not observed by XMM-Newton), and of the diffuse soft X-ray emission in the vicinity of the Hercules concentration.

\section{ROSAT PSPC data of the Hercules supercluster}

The ROSAT PSPC instrument features a large field of view ( $\sim 1$ degree radius), an effective area of $\sim 200 \mathrm{~cm}^{2}$ at $0.25 \mathrm{keV}$, negligible low-energy particle background (Plucinsky et al. 1993) and stable calibration. Moreover, a large number of observations are available in the public archive that sample the diffuse emission in the neighborhood of the Hercules supercluster. These characteristics render the PSPC optimally suited for the study of low-energy emission from diffuse structures, such as galaxy clusters and their surroundings, and of the diffuse soft X-ray background. A set of prescriptions for the study
Table 1. PSPC observations.

\begin{tabular}{lcc}
\hline \hline Pointing & Archival ID & ${\text { Time }(\mathrm{ks})^{a}}^{{ }^{a}}$ \\
\hline MKW03s & RP800128 & 7.8 \\
A2052 & RP800275 & 5.2 \\
A2063 & RP800184 & 8.4 \\
Background 1 & RP701001 & 8.1 \\
Background 2 & RP400117 & 6.6 \\
Background 3 & RP700257 & 16.1 \\
Background 4 & RP200954 & 3.0 \\
Background 5 & RP600257 & 6.8 \\
Background 6 & RP200532 & 5.2 \\
Background 7 & RP700897 & 7.9 \\
Background 8 & RP200510 & 14.0 \\
\hline
\end{tabular}

${ }^{a}$ Exposure times are effective exposures after screening of high background intervals and application of good-time interval filters.

of diffuse emission with the PSPC is provided by Snowden et al. (1994); we followed their recipes, and a detailed description of our data reduction methods is provided in Bonamente et al. (2002) and in Bonamente et al. (2003).

We analyzed 11 pointed observations of the southern region of the Hercules supercluster. Three of these observations are centered on the clusters MKW03s, A2052 and A2063, and the remaining 8 pointings span an area of $\sim 20$ degree radius around the three clusters, and are used for study of the background. A summary of the PSPC observations is in Table 1. In Fig. 1 we show a layout of the observations, overlaid on a $R 2$ $(\sim 0.15-0.3 \mathrm{keV})$ band image from the ROSAT All-Sky Survey (RASS).

\section{The north polar spur region}

The three clusters under investigation (MKW03s, A2052 and A2063) lie in a region of the sky known as the North Polar Spur (NPS), or Radio Loop 1. It is evident from Fig. 1, which covers only a southern extension of that region, that the NPS features enhanced soft X-ray emission with respect to the surroundings. The enhancement is in the approximate shape of an arc that rises from $l \sim 30 \mathrm{deg}, b \sim 40 \mathrm{deg}$ towards the North Pole, and then descends back to lower Galactic latitudes (not shown in Fig. 1). The $R 2$ band maps from the RASS in fact reveal that the NPS is the brightest feature in the whole soft X-ray sky. Due to its approximately circular shape, the NPS is commonly interpreted as the remnant of a nearby Galactic supernova explosion (e.g., Iwan 1980). An extragalactic origin of all or part of the soft X-ray emissiion from the NPS cannot however be excluded. The Kuntz et al. (2001) study of the Galactic North Polar cap concluded that its $T \sim 10^{6.5} \mathrm{~K}$ emission could either be due to a Galactic halo or to a diffuse extragalactic component. At lower Galactic latitudes along the NPS, Kaastra et al. (2003a) found that the $0.2 \mathrm{keV}$ soft X-ray emission in the direction of MKW03s and A2052 is spectrally similar, and 6 times brighter, to the Kuntz et al. (2001) emission in the North Polar cap. Large scale cosmological simulations (e.g., Cen \& Ostriker 1999; Davè et al. 2001) and observations 


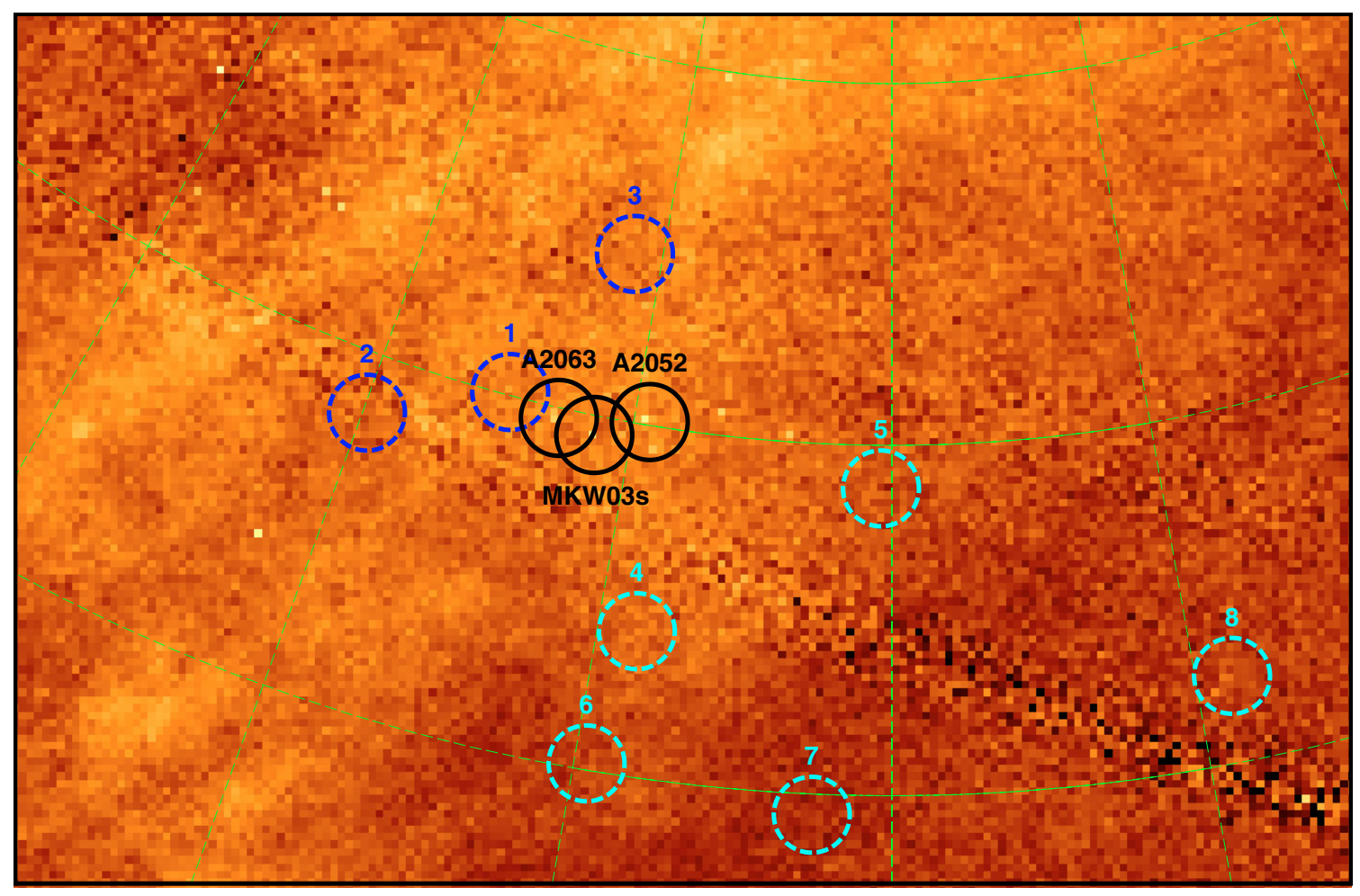

Fig. 1. PSPC observations towards the Hercules supercluster, overlaid on an RASS $R 2(0.15-0.3 \mathrm{keV})$ band image. The image is in Galactic coordinates, latitude lines are respectively 40,50 and 60 degrees North, longitude lines are $-30,-20,-10,0,+10$ and +20 degrees. The southern portion of the North polar Spur is the bright region in lighter colors.

of absorption line systems (e.g., Tripp et al. 2000; Oegerle et al. 2000; Tripp \& Savage 2000; Savage et al. 2002; Sembach et al. 2001; Shull et al. 2003) indicate that large amounts of intergalactic warm gas at $T \sim 10^{6} \mathrm{~K}$ are ubiquitous in the nearby universe. It is therefore possible to consider that part of the soft X-ray enhancement in the NPS is of extragalactic origin. A conclusive determination of Galactic or extra-galactic origin of the NPS emission requires high-throughput X-ray spectroscopy with electronvolt resolution.

The 11 fields of Table 1 were analyzed in order to assess the level of inhomogenieties of the soft X-ray emission around the clusters MKW03s, A2052 and A2063. The 8 background fields were randomly chosen among the PSPC observations available in that region, and are representative of the local X-ray emission. All point sources and diffuse emission regions were excluded from the fields, and the data divided into three bands following Snowden's convention (Snowden et al. 1997): $R 2$ band ( $\sim 0.15-0.3 \mathrm{keV}), R 4$ band $(\sim 0.4-1.0 \mathrm{keV})$ and $R 7$ band $(\sim 1.1-2.0 \mathrm{keV})^{1}$. The $R 2$ band, or $1 / 4 \mathrm{keV}$ band, is the softest PSPC band that has stable response and reliable calibration, $R 4$ comprises the spectral region where high-ionization oxygen lines (e.g., OVII) may appear, and $R 7$ is PSPC's hardest band, the most sensitive to the hot ICM emission.

In Table 2 we show the $R 2, R 4$ and $R 7$ band background levels for our 11 fields. The background was measured from the $30-50^{\prime}$ region for the three cluster observations (see Sect. 5.1)

1 This choice is also dictated by the availability of calibration data and software tools that use this band convention. and from the entire field of view for the other background observations. All bright point sources were excluded. The 3 cluster fields and background fields 1, 2 and 3 are in the general sky region where the NPS enhancement is more pronounced, while background fields $4,5,6,7$ and 8 are further away from the cluster fields, in a region of the sky where the NPS enhancement is less evident. The $R 2$ band background levels have an average of $\mu_{R 2}=5.9 \times 10^{-4}$ counts s$^{-1}$ arcmin $^{-2}$, with a standard deviation of $\sigma_{R 2}=31 \%$ among the 11 fields. The $R 4$ band average flux is $\mu_{R 4}=2.4 \times 10^{-4}$ counts s$^{-1} \operatorname{arcmin}^{-2}$, with $\sigma_{R 4}=$ $81 \%$, and in the $R 7$ band $\mu_{R 7}=0.61 \times 10^{-4}$ counts s$^{-1}$ arcmin $^{-2}$ and $\sigma_{R 7}=15 \%$. The $R 2$ band inhomogenieties are consistent with those assumed by Kaastra et al. (2003a,b) in their analysis of XMM data. Fluctuations of the $R 4$ band fluxes are considerably more pronounced, and need to be accounted for when trying to detect emission features that are close to the background level. We will return on this point in Sect. 5.4.

\section{The soft excess emission in MKW03s, A2052 and A2063}

We start by obtaining radial profiles of the surface brightness of the three clusters' diffuse emission, divided in the three bands $R 2, R 4$ and $R 7$ (Figs. 2, 3 and 4). The $R 7$ emission is dominated by the cluster hot ICM, and the hot ICM emission extends out to $\sim 15$ arcmin for MKW03s and 20 arcmin for A2052 and A2063. The soft emission ( $R 2$ band) is less centrally peaked than the harder $R 7$ emission, and it flattens out to a constant level a few arcmin earlier than the corresponding $R 7$ radial 
Table 2. Background levels.

\begin{tabular}{lccc}
\hline \hline Pointing & $R 2$ band c/r & $\begin{array}{c}R 4 \text { band c/r } \\
\left(\text { counts s}^{-1} \mathrm{arcmin}^{-2}\right)\end{array}$ & $R 7 \mathrm{band} \mathrm{c/r^{a }}$ \\
\hline MKw03s & $5.20 \pm 0.10 \times 10^{-4}$ & $1.30 \pm 0.05 \times 10^{-4}$ & $0.61 \pm 0.04 \times 10^{-4}$ \\
A2052 & $6.24 \pm 0.13 \times 10^{-4}$ & $1.44 \pm 0.07 \times 10^{-4}$ & $0.67 \pm 0.05 \times 10^{-4}$ \\
A2063 & $6.32 \pm 0.11 \times 10^{-4}$ & $1.55 \pm 0.05 \times 10^{-4}$ & $0.71 \pm 0.04 \times 10^{-4}$ \\
Background 1 & $6.60 \pm 0.10 \times 10^{-4}$ & $1.57 \pm 0.06 \times 10^{-4}$ & $0.59 \pm 0.04 \times 10^{-4}$ \\
Background 2 & $7.65 \pm 0.12 \times 10^{-4}$ & $4.51 \pm 0.10 \times 10^{-4}$ & $0.56 \pm 0.03 \times 10^{-4}$ \\
Background 3 & $9.68 \pm 0.10 \times 10^{-4}$ & $5.89 \pm 0.09 \times 10^{-4}$ & $0.73 \pm 0.03 \times 10^{-4}$ \\
\hline Background 4 & $4.72 \pm 0.14 \times 10^{-4}$ & $1.09 \pm 0.07 \times 10^{-4}$ & $0.50 \pm 0.06 \times 10^{-4}$ \\
Background 5 & $5.26 \pm 0.10 \times 10^{-4}$ & $1.24 \pm 0.04 \times 10^{-4}$ & $0.52 \pm 0.04 \times 10^{-4}$ \\
Background 6 & $3.23 \pm 0.09 \times 10^{-4}$ & $1.25 \pm 0.07 \times 10^{-4}$ & $0.63 \pm 0.04 \times 10^{-4}$ \\
Background 7 & $3.44 \pm 0.08 \times 10^{-4}$ & $0.95 \pm 0.04 \times 10^{-4}$ & $0.49 \pm 0.03 \times 10^{-4}$ \\
Background 8 & $6.41 \pm 0.09 \times 10^{-4}$ & $5.89 \pm 0.09 \times 10^{-4}$ & $0.73 \pm 0.03 \times 10^{-4}$ \\
\hline Average & $5.9 \times 10^{-4}$ & $2.4 \times 10^{-4}$ & $0.61 \times 10^{-4}$ \\
Standard deviation & $31 \%$ & $81 \%$ & $15 \%$ \\
\hline
\end{tabular}

${ }^{a}$ Errors are $90 \%$ confidence intervals.

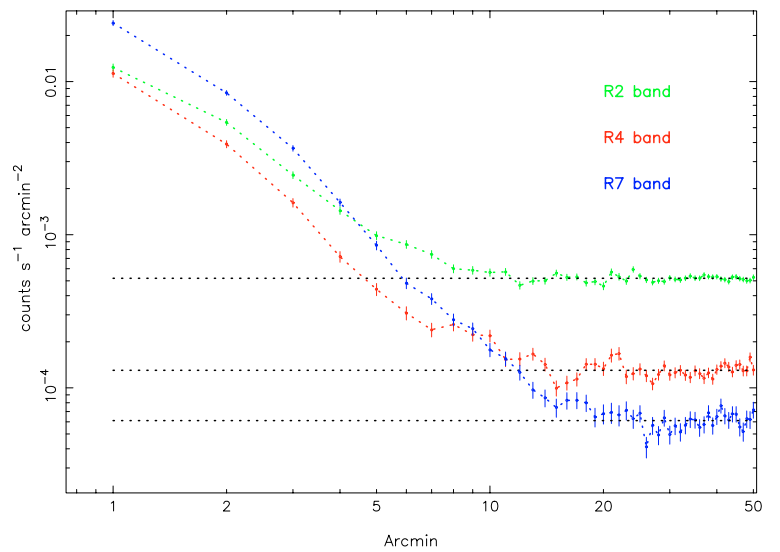

Fig. 2. Radial profile of surface brightness for cluster MKW03s in the $R 2, R 4$ and $R 7$ bands. Dotted lines are the background levels as obtained from a fit to the $30-50^{\prime}$ data.

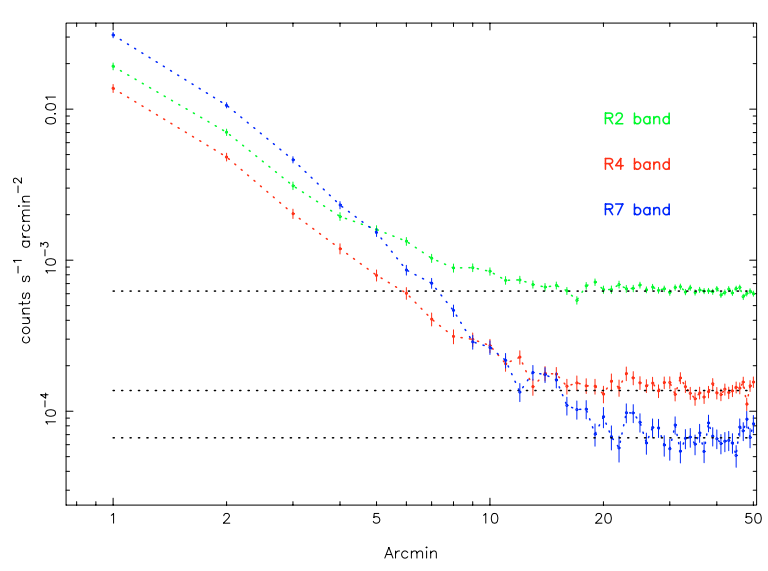

Fig. 3. Same as Fig. 2, but for cluster A2052.

profile. We therefore use the cluster-free $30-50^{\prime}$ region to measure the local background in the 3 cluster observations.

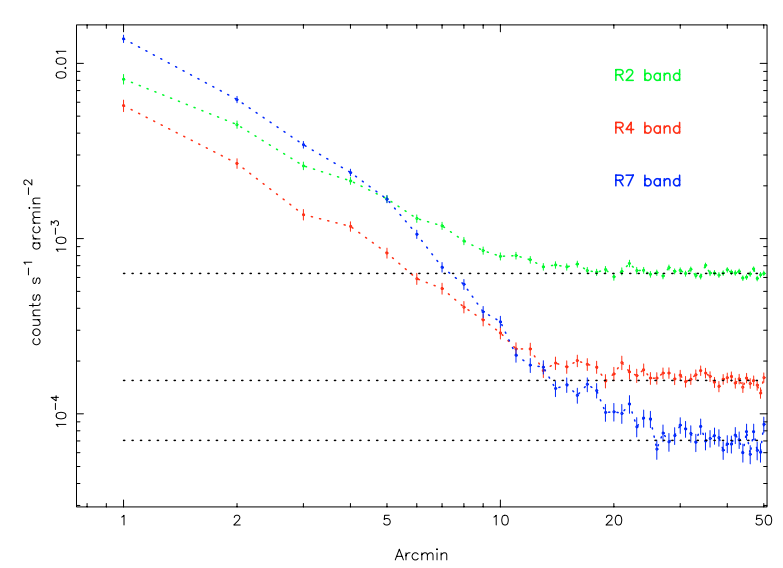

Fig. 4. Same as Fig. 2, but for cluster A2063.

\subsection{Spectral analysis with local background}

In order to test the existence of soft excess, we proceed with the spectral analysis of the cluster data. Each cluster is divided into 5 annuli out to a radius of 18 arcmin. Data between $\sim 18-25^{\prime}$ from boresight are affected by obscuration from the PSPC support structure, and are not considered.

In earlier studies of the soft excess emission with the PSPC instrument (e.g., Bonamente et al. 2002) we always employed a local background for the purpose of background subtraction. Given PSPC's large field of view, peripheral regions of each cluster pointing are free of cluster emission, and can therefore be used for determining the background levels. For each cluster, we use a region $30-50^{\prime}$ from boresight to extract a background spectrum, which is then applied to the inner annuli. As the PSPC instrument has a response that declines with offset angle (vignetting effect), the background spectra are properly rescaled before being used for background subtraction in the inner annuli. See Bonamente et al. (2002) for further details on the background subtraction method. 
Table 3. Spectral analysis with local background subtraction.

\begin{tabular}{|c|c|c|c|c|c|c|c|}
\hline \multirow{3}{*}{$\begin{array}{l}\text { Region } \\
\text { (arcmin) }\end{array}$} & \multirow{3}{*}{$\begin{array}{c}k T \\
(\mathrm{keV})\end{array}$} & \multirow{3}{*}{$\begin{array}{c}A \\
\text { (solar) }\end{array}$} & \multirow[t]{3}{*}{$\chi^{2}$ (d.o.f.) } & \multicolumn{2}{|r|}{$R 2$} & \multicolumn{2}{|r|}{$R 4$} \\
\hline & & & & Detected & predicted & detected & predicted \\
\hline & & & & \multicolumn{4}{|c|}{ (count/s) } \\
\hline \multicolumn{8}{|c|}{ MKW03s } \\
\hline $0-2$ & $3.3 \pm 0.4$ & 0.6 & $69.6(70)$ & $0.102 \pm 0.004$ & $0.100 \pm 0.003 \pm 0.002 \pm 0.005$ & $0.087 \pm 0.004$ & $0.088 \pm 0.002 \pm 0.002$ \\
\hline $2-4$ & 3 & 0.4 & $60.1(55)$ & $0.062 \pm 0.003$ & $0.058 \pm_{0.003}^{0.005} \pm 0.002 \pm 0.003$ & $0.044 \pm 0.003$ & $0.050 \pm 0.002 \pm_{0.002}^{0.001}$ \\
\hline $4-8$ & $2.1 \pm_{0.3}^{0.4}$ & 0.2 & $40.4(43)$ & $0.051 \pm 0.004$ & $0.050 \pm 0.001 \pm 0.001 \pm 0.003$ & $0.030 \pm 0.003$ & $0.039 \pm 0.001 \pm_{0.001}^{0.002}$ \\
\hline $8-12$ & 2.5 & 0.1 & 20.1(26) & $0.009 \pm 0.005$ & $0.022 \pm_{0.001}^{0.002} \pm 0.001 \pm 0.001$ & $0.016 \pm 0.003$ & $0.017 \pm 0.001 \pm 0.001$ \\
\hline $12-18$ & 2.5 & 0.1 & $36.2(29)$ & $0.006 \pm 0.007$ & $0.009 \pm 0.001 \pm 0.001 \pm 0.001$ & $-0.003 \pm 0.003$ & $0.007 \pm 0.001 \pm 0.001$ \\
\hline \multicolumn{8}{|c|}{ A2052 } \\
\hline $0-2$ & $2.3 \pm_{0.2}^{0.3}$ & 0.6 & $67.5(63)$ & $0.134 \pm 0.005$ & $0.127 \pm 0.003 \pm_{0.001}^{0.002} \pm 0.006$ & $0.101 \pm 0.004$ & $0.110 \pm 0.002 \pm_{0.003}^{0.004}$ \\
\hline $2-4$ & $3.2 \pm_{0.5}^{0.8}$ & 0.4 & $54.3(44)$ & $0.082 \pm 0.005$ & $0.081 \pm_{0.004}^{0.002} \pm 0.001 \pm 0.004$ & $0.057 \pm 0.003$ & $0.069 \pm_{0.002}^{0.001} \pm 0.002$ \\
\hline $4-8$ & $2.7 \pm_{0.4}^{0.7}$ & 0.4 & $48.1(47)$ & $0.094 \pm 0.006$ & $0.074 \pm_{0.003}^{0.002} \pm 0.001 \pm 0.004$ & $0.063 \pm 0.004$ & $0.062 \pm 0.001 \pm_{0.001}^{0.002}$ \\
\hline $8-12$ & $2.9 \pm_{0.9}^{3.4}$ & 0.2 & $7.6(22)$ & $0.049 \pm 0.007$ & $0.029 \pm_{0.004}^{0.002} \pm 0.001 \pm 0.002$ & $0.031 \pm 0.004$ & $0.024 \pm_{0.002}^{0.001} \pm 0.001$ \\
\hline $12-18$ & 2.5 & 0.2 & $25.2(26)$ & $0.009 \pm 0.009$ & $0.024 \pm_{0.001}^{0.002} \pm 0.001 \pm 0.001$ & $0.012 \pm 0.005$ & $0.019 \pm 0.001 \pm 0.001$ \\
\hline \multicolumn{8}{|c|}{ A2063 } \\
\hline $0-2$ & $3.2 \pm_{0.5}^{0.7}$ & 0.4 & $67.2(55)$ & $0.069 \pm 0.003$ & $0.059 \pm 0.002 \pm 0.001 \pm 0.003$ & $0.049 \pm 0.002$ & $0.053 \pm 0.001 \pm_{0.002}^{0.001}$ \\
\hline $2-4$ & $3.9 \pm_{0.7}^{1.0}$ & 0.4 & $55.9(58)$ & $0.072 \pm 0.003$ & $0.061 \pm 0.003 \pm 0.001 \pm 0.003$ & $0.045 \pm 0.002$ & $0.055 \pm_{0.002}^{0.001} \pm 0.001$ \\
\hline $4-8$ & $2.7 \pm 0.4$ & 0.3 & $65.4(66)$ & $0.104 \pm 0.005$ & $0.079 \pm_{0.002}^{0.003} \pm 0.001 \pm 0.004$ & $0.069 \pm 0.003$ & $0.070 \pm 0.001 \pm 0.002$ \\
\hline $8-12$ & $2.2 \pm_{0.4}^{0.6}$ & 0.3 & $40.5(43)$ & $0.048 \pm 0.005$ & $0.035 \pm_{0.002}^{0.001} \pm 0.001 \pm 0.002$ & $0.033 \pm 0.003$ & $0.029 \pm 0.001 \pm 0.001$ \\
\hline $12-18$ & 2 & 0.3 & $39.8(43)$ & $0.034 \pm 0.007$ & $0.024 \pm_{0.001}^{0.003} \pm 0.001 \pm 0.001$ & $0.022 \pm 0.004$ & $0.020 \pm_{0.001}^{0.008} \pm 0.001$ \\
\hline
\end{tabular}

Errors in predicted fluxes are respectively those due to $\Delta k T, \Delta A$ and $\Delta N_{\mathrm{H}} . A$ is elemental abundances as fraction of Solar. Uncertainties due to $\Delta N_{\mathrm{H}}$ in $\mathrm{R} 4$ band are not shown, as smaller than the employed numerical accuracy.

Errors are 1- $\sigma$ uncertainties (68\% confidence levels). All errors are added in quadrature, except those relative to $\Delta N_{\mathrm{H}}$, which are added linearly as a possible systematic error.

First we determine the temperature of the hot ICM plasma, by fitting the $\sim 1-2 \mathrm{keV}$ data alone (pulse invariant channels $\sim 100-200)$ to a photoabsorbed optically-thin plasma model (WABS and MEKAL models in XSPEC). The $\sim 1-2 \mathrm{keV}$ band is in fact dominated by the emission from the hot ICM, and any potential soft excess radiation is marginal in this band. This method of fitting the high energy data alone, instead of the full data, is the same we adopted in earlier publications (e.g., Bonamente et al. 2003), and it is commonly done for the study of the soft excess in AGNs (e.g., Page et al. 2004; Brinkmann et al. 2003). The best-fit temperatures we derived with this method are in agreement with measurements of other instruments with larger passband, such as ASCA and XMM, even for high temperature clusters (Bonamente et al. 2003; Bonamente et al. 2002). The energy resolution of the PSPC instrument is $\frac{\Delta(E)}{E} \sim 0.43\left(\frac{E}{0.93 \mathrm{keV}}\right)^{-0.5} F W H M$, which yields a value of $\frac{\Delta(E)}{E} \sim 0.34$ at $E=1.5 \mathrm{keV}$. The $1-2 \mathrm{keV}$ band therefore covers several independent energy bands (approximately bands $R 5, R 6$ and $R 7$ following the convention of Snowden et al. 1993).

The hot plasma temperature of the three clusters is generally in the $2-4 \mathrm{keV}$ range, and our PSPC data can constrain the temperature to within $\leq 25 \%$ uncertainty for most regions (see Table 3). In some regions of MKW03s and A2052 with limited $\mathrm{S} / \mathrm{N}$ we use the temperatures measured by XMM
(Kaastra et al. 2004) and use a conservative estimate for the temperature uncertainty of $\pm 1 \mathrm{keV}$ in the error analysis that follows (these regions are shown without error in Table 3). In the outermost annulus of A2063, for which there is no available XMM data, we assume a temperature of $k T=2 \pm 1 \mathrm{keV}$, according to the temperature decrease at large radii generally observed in galaxy clusters (e.g., De Grandi \& Molendi 2002).

When the hot ICM temperature is fitted to the PSPC data, tha best-fit values are in agreement with the single-temperature results from XMM data (Kaastra et al. 2004). Metal abundances are fixed at the vaules measured by other instruments (Tamura et al. 2004; Mushotzky et al. 1996; Blanton et al. 2003; Finoguenov et al. 2001), and a fiducial uncertainty of \pm 0.1 solar is used in the error analysis. Galactic absorption is fixed at the measurements of Dickey \& Lockman (1990), which are $N_{\mathrm{H}}=2.9 \times 10^{20} \mathrm{~cm}^{-2}$ for MKW03s and A2052 and $N_{\mathrm{H}}=3.0 \times 10^{20} \mathrm{~cm}^{-2}$ for A2063, and the formal uncertainty of $\Delta N_{\mathrm{H}}=1 \times 10^{19}$ is used in the error analysis.

Gas in the central regions $(\leq 2$ arcmin from the cluster center) may be multiphase, due to the effect of radiative cooling (e.g., Peterson et al. 2003). XMM data of Kaastra et al. (2004) indicate that the MKW03s spectra are consistent with a single-phase thermal spectrum at all radii covered by $\mathrm{XMM}\left(\leq 12^{\prime}\right)$, and at radii $\geq 1$ arcmin for A2052. We contend that a single-temperature model of PSPC spectra may not be 


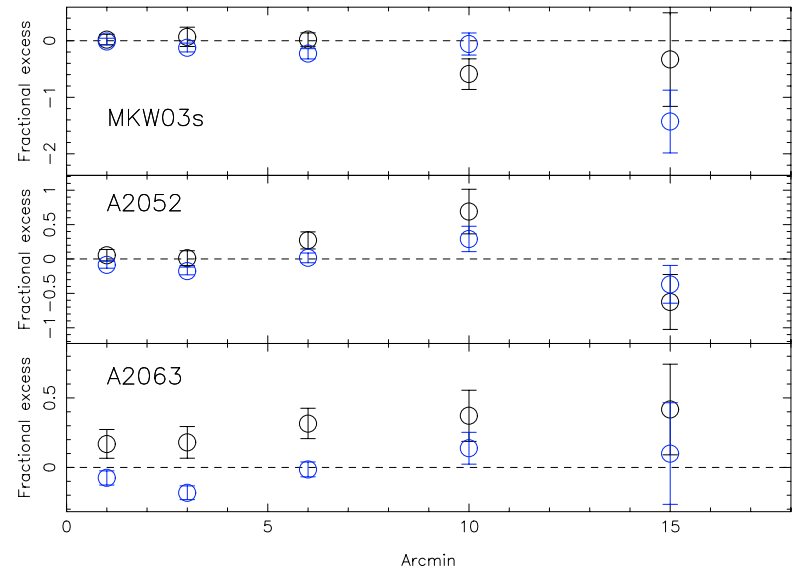

Fig. 5. Fractional excess in $R 2$ band (black) and in $R 4$ band (blue) for the three clusters (from top to bottom: MKS03s, A2052 and A2062) using local background.

appropriate only in the innermost annulus of A2052 and, possibly, of A2063, for which there are no available XMM data. Since there is no evidence of soft excess emission in these inner radii irrespective of the background subtraction method, see following, we do not employ multi-phase models for the hot ICM of the 3 galaxy clusters.

Results of the spectral fits are shown in Table 3. Once a best-fit model to the hot ICM is in place, we are able to predict the hot ICM emission in the lower energy $R 2$ and $R 4$ bands, and compare predictions with measured fluxes, also shown in Table 3. In Fig. 5 we also plot the fractional excess emission $\eta$, defined as $\eta=\frac{O-P}{P}$, where $O$ is the detected $R 2$ or $R 4$ band flux, and $P$ is the corresponding model prediction according to the thermal models of Table 3. Uncertanties in the hot ICM temperature and abundance, in the Galactic H column density and in the detected fluxes are properly accounted for in the determination of $\sigma_{\eta}$.

Figure 5 shows that little or no excess emission is detected in the MKW03s data when this local background is employed, while A2052 and A2063 show some excess emission preferentially distributed in the softest channels and at large radii. The statistical significance of the $R 2$ band soft excess detection is $\sim 3 \sigma$ for $\mathrm{A} 2052$, and $\sim 4.5 \sigma$ for $\mathrm{A} 2063$.

\subsection{Spectral analysis with other backgrounds}

We also employ the other 8 fields in Table 1 for the purpose of background subtraction. Fields 1-3 are located in the NPS region, at a distance of $\sim 1-10$ degrees from the clusters ("nearby" fields), fields 4-8 lie outside the NPS, at a distance of 10-20 degrees from the clusters ("distant" fields).

For each of the 8 pointings a spectrum is extracted after exclusion of all point-like or diffuse emission features. The spectrum is then used as a background for the cluster annular regions (see Sect. 5.1). A different background than the local one employed in Sect. 5.1 results in different soft excess fluxes, but leaves the hot ICM parameters $k T$ and $A$ virtually unchanged. This is due to the fact that the $1-2 \mathrm{keV}$ background has little variability (Table 2; see also Snowden et al. 1995). In Table 4

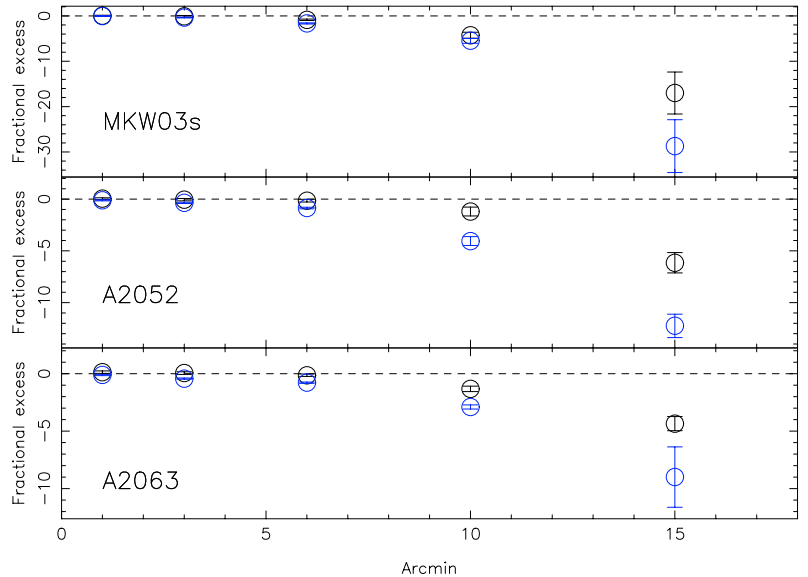

Fig. 6. Same as Fig. 5, but using nearby background field 3.

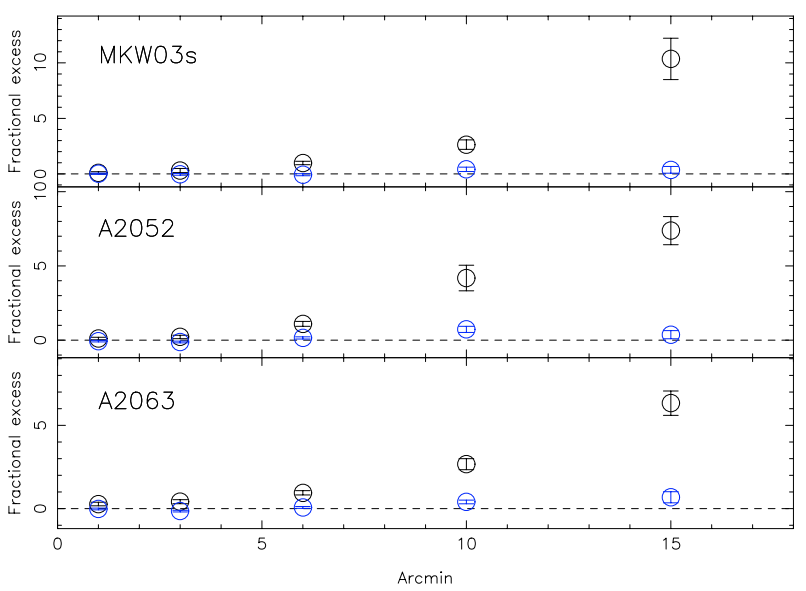

Fig. 7. Same as Fig. 5, but using distant background field 6.

we show the detected and predicted $R 2$ and $R 4$ band count rates, where the relevant hot ICM parameters $k T$ and $A$ are the same as in Table 3.

The fractional excess plots of Figs. 6 and 7 show how strongly the choice of the background impacts the determination of soft excess fluxes. The use of a nearby background (Fig. 6) yields an oversubtraction of soft X-rays, while the use of a distant background (Fig. 7) results in a significant soft excess detection, similar to the XMM results. Background-subtracted fluxes using all background fields are shown in Table 4. In Fig. 8 we show the PSPC spectra of background field 3 (representative of the nearby background), background field 6 (representative of distant background fields) and the local background from the A2052 observation. These backgrounds are applied to a representative region of A2052 (8-12' annulus) and the corresponding background subtracted spectra are shown in Fig. 9. The background spectra differ mostly at low energy ( $E \leq 1 \mathrm{keV})$, as also shown in Table 2, with the local and nearby background spectra featuring stronger emission than the distant field. Using the distant background instead of an in situ background will result in an overestimate of the cluster soft excess fluxes and, in instruments of higher spectral resulution such as the XMM detectors, in spurious detections of emission features at $E \simeq 0.5 \mathrm{keV}$ that mimick OVII lines ( $E=0.560 \mathrm{keV}$ in rest frame; Kaastra et al. 2003). 
Table 4. Spectral analysis with other backgrounds.

\begin{tabular}{|c|c|c|c|c|c|c|c|c|c|c|c|c|}
\hline \multirow{4}{*}{$\begin{array}{l}\text { Region } \\
\text { (arcmin) } \\
0-21\end{array}$} & \multicolumn{4}{|c|}{ MKW03s } & \multicolumn{4}{|c|}{ A2052 } & \multicolumn{4}{|c|}{ A2063 } \\
\hline & \multicolumn{2}{|c|}{$R 2$} & \multicolumn{2}{|c|}{$R 4$} & \multicolumn{2}{|c|}{$R 2$} & \multicolumn{2}{|c|}{$R 4$} & \multicolumn{2}{|c|}{$R 2$} & \multicolumn{2}{|c|}{$R 4$} \\
\hline & detected & predicted & detected & predicted & detected & $\begin{array}{r}\text { predicted } \\
\text { (co }\end{array}$ & $\begin{array}{l}\text { detected } \\
\text { t/s) }\end{array}$ & predicted & detected & predicted & detected & predicted \\
\hline & 0.100 & 0.095 & 0.087 & 0.088 & 0.134 & 0.125 & 0.100 & 0.108 & 0.068 & 0.058 & 0.049 & 0.051 \\
\hline 2 & 0.098 & 0.094 & 0.083 & 0.085 & 0.132 & 0.125 & 0.096 & 0.108 & 0.066 & 0.058 & 0.045 & 0.051 \\
\hline 3 & 0.098 & 0.094 & 0.083 & 0.085 & 0.131 & 0.125 & 0.096 & 0.108 & 0.066 & 0.058 & 0.045 & 0.051 \\
\hline 4 & 0.103 & 0.096 & 0.088 & 0.087 & 0.136 & 0.125 & 0.101 & 0.108 & 0.071 & 0.058 & 0.050 & 0.051 \\
\hline 5 & 0.102 & 0.096 & 0.087 & 0.087 & 0.136 & 0.125 & 0.101 & 0.108 & 0.070 & 0.058 & 0.050 & 0.051 \\
\hline 6 & 0.105 & 0.096 & 0.088 & 0.087 & 0.139 & 0.125 & 0.101 & 0.108 & 0.074 & 0.058 & 0.050 & 0.051 \\
\hline 7 & 0.105 & 0.096 & 0.088 & 0.087 & 0.139 & 0.125 & 0.101 & 0.108 & 0.073 & 0.058 & 0.050 & 0.051 \\
\hline 8 & 0.102 & 0.094 & 0.082 & 0.086 & 0.135 & 0.125 & 0.096 & 0.108 & 0.070 & 0.058 & 0.044 & 0.051 \\
\hline $2-41$ & 0.056 & 0.055 & 0.044 & 0.047 & 0.080 & 0.077 & 0.056 & 0.066 & 0.072 & 0.060 & 0.045 & 0.054 \\
\hline 2 & 0.051 & 0.056 & 0.031 & 0.047 & 0.074 & 0.077 & 0.043 & 0.066 & 0.064 & 0.060 & 0.032 & 0.054 \\
\hline 3 & 0.051 & 0.056 & 0.030 & 0.047 & 0.073 & 0.077 & 0.042 & 0.066 & 0.063 & 0.060 & 0.031 & 0.054 \\
\hline 4 & 0.065 & 0.056 & 0.046 & 0.047 & 0.087 & 0.078 & 0.058 & 0.066 & 0.077 & 0.060 & 0.046 & 0.054 \\
\hline 5 & 0.063 & 0.056 & 0.045 & 0.048 & 0.086 & 0.077 & 0.057 & 0.066 & 0.075 & 0.060 & 0.046 & 0.054 \\
\hline 6 & 0.073 & 0.056 & 0.046 & 0.048 & 0.095 & 0.078 & 0.058 & 0.066 & 0.085 & 0.060 & 0.046 & 0.054 \\
\hline 7 & 0.071 & 0.056 & 0.046 & 0.048 & 0.093 & 0.078 & 0.058 & 0.066 & 0.083 & 0.060 & 0.047 & 0.054 \\
\hline 8 & 0.061 & 0.056 & 0.030 & 0.048 & 0.084 & 0.077 & 0.042 & 0.066 & 0.074 & 0.060 & 0.030 & 0.054 \\
\hline $4-81$ & 0.035 & 0.044 & 0.029 & 0.035 & 0.087 & 0.069 & 0.062 & 0.058 & 0.095 & 0.079 & 0.068 & 0.069 \\
\hline 2 & 0.009 & 0.047 & -0.023 & 0.037 & 0.062 & 0.070 & 0.010 & 0.059 & 0.072 & 0.079 & 0.016 & 0.069 \\
\hline 3 & 0.007 & 0.047 & -0.024 & 0.037 & 0.059 & 0.069 & 0.008 & 0.058 & 0.068 & 0.078 & 0.015 & 0.069 \\
\hline 4 & 0.062 & 0.049 & 0.035 & 0.039 & 0.115 & 0.071 & 0.067 & 0.060 & 0.121 & 0.080 & 0.074 & 0.070 \\
\hline 5 & 0.055 & 0.048 & 0.033 & 0.038 & 0.108 & 0.070 & 0.066 & 0.060 & 0.116 & 0.079 & 0.072 & 0.069 \\
\hline 6 & 0.095 & 0.048 & 0.035 & 0.038 & 0.147 & 0.070 & 0.068 & 0.059 & 0.156 & 0.080 & 0.075 & 0.070 \\
\hline 7 & 0.087 & 0.049 & 0.038 & 0.039 & 0.140 & 0.071 & 0.071 & 0.060 & 0.147 & 0.080 & 0.077 & 0.070 \\
\hline 8 & 0.052 & 0.048 & -0.027 & 0.038 & 0.105 & 0.070 & 0.005 & 0.059 & 0.112 & 0.079 & 0.012 & 0.069 \\
\hline $8-121$ & -0.019 & 0.020 & 0.014 & 0.016 & 0.039 & 0.025 & 0.027 & 0.021 & 0.034 & 0.034 & 0.032 & 0.029 \\
\hline 2 & -0.058 & 0.020 & -0.069 & 0.016 & -0.001 & 0.025 & -0.058 & 0.020 & -0.008 & 0.035 & -0.052 & 0.029 \\
\hline 3 & -0.065 & 0.020 & -0.072 & 0.016 & -0.005 & 0.025 & -0.061 & 0.020 & -0.011 & 0.034 & -0.055 & 0.029 \\
\hline 4 & 0.024 & 0.023 & 0.024 & 0.018 & 0.085 & 0.027 & 0.038 & 0.022 & 0.076 & 0.036 & 0.042 & 0.030 \\
\hline 5 & 0.014 & 0.021 & 0.021 & 0.016 & 0.074 & 0.026 & 0.034 & 0.021 & 0.067 & 0.035 & 0.039 & 0.030 \\
\hline 6 & 0.080 & 0.022 & 0.024 & 0.017 & 0.140 & 0.027 & 0.038 & 0.022 & 0.132 & 0.036 & 0.042 & 0.030 \\
\hline 7 & 0.066 & 0.023 & 0.030 & 0.018 & 0.126 & 0.028 & 0.042 & 0.023 & 0.118 & 0.037 & 0.047 & 0.031 \\
\hline 8 & 0.010 & 0.021 & -0.077 & 0.016 & 0.069 & 0.025 & -0.065 & 0.021 & 0.062 & 0.035 & -0.059 & 0.030 \\
\hline $12-181$ & -0.048 & 0.010 & -0.007 & 0.008 & -0.006 & 0.020 & 0.006 & 0.016 & 0.010 & 0.026 & 0.019 & 0.022 \\
\hline 2 & -0.133 & 0.010 & -0.186 & 0.008 & -0.090 & 0.020 & -0.173 & 0.016 & -0.080 & 0.026 & -0.160 & 0.022 \\
\hline 3 & -0.144 & 0.009 & -0.194 & 0.007 & -0.103 & 0.020 & -0.180 & 0.016 & -0.087 & 0.026 & -0.168 & 0.021 \\
\hline 4 & 0.044 & 0.014 & 0.013 & 0.011 & 0.083 & 0.025 & 0.024 & 0.020 & 0.103 & 0.03 & 0.042 & 0.025 \\
\hline 5 & 0.021 & 0.011 & 0.006 & 0.009 & 0.064 & 0.022 & 0.019 & 0.018 & 0.081 & 0.028 & 0.033 & 0.024 \\
\hline 6 & 0.159 & 0.014 & 0.015 & 0.011 & 0.201 & 0.024 & 0.026 & 0.019 & 0.220 & 0.030 & 0.042 & 0.025 \\
\hline 7 & 0.132 & 0.017 & 0.026 & 0.013 & 0.173 & 0.027 & 0.036 & 0.022 & 0.189 & 0.033 & 0.051 & 0.027 \\
\hline 8 & 0.009 & 0.011 & -0.203 & 0.009 & 0.050 & 0.022 & -0.190 & 0.017 & 0.069 & 0.028 & -0.177 & 0.023 \\
\hline
\end{tabular}

Errors in the detected fluxes and in the predicted fluxes are virtually identical to those of Table 3, and therefore not shown here for clarity. Numerals in the regions column label the background used.

\subsection{Choice of background}

The choice of background affects the measurement of the cluster soft X-ray fluxes (see Fig. 9). In this section we discuss the applicability of the three kinds of background (local, nearby and distant) discussed in Sects. 5.1 and 5.2 considering three possible scenarios: (a) the NPS soft X-ray enhancement is of Galactic origin, (b) of extragalactic origin and (c) in part Galactic and in part extragalactic.

(a) All of the enhanced soft X-ray emission in the NPS region (see Fig. 1) is of Galactic origin, and as such should be 


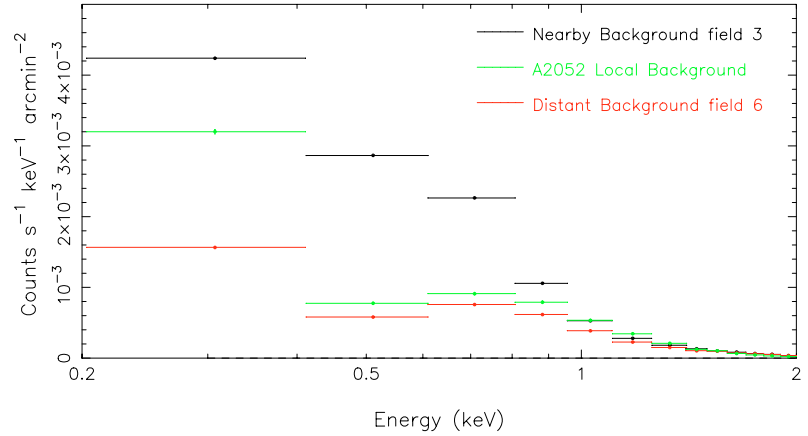

Fig. 8. PSPC spectra of three representative background regions. The spectra were rebinned to a resolution of $\sim 1 / 3 F W H M$ of the PSPC energy resolution.

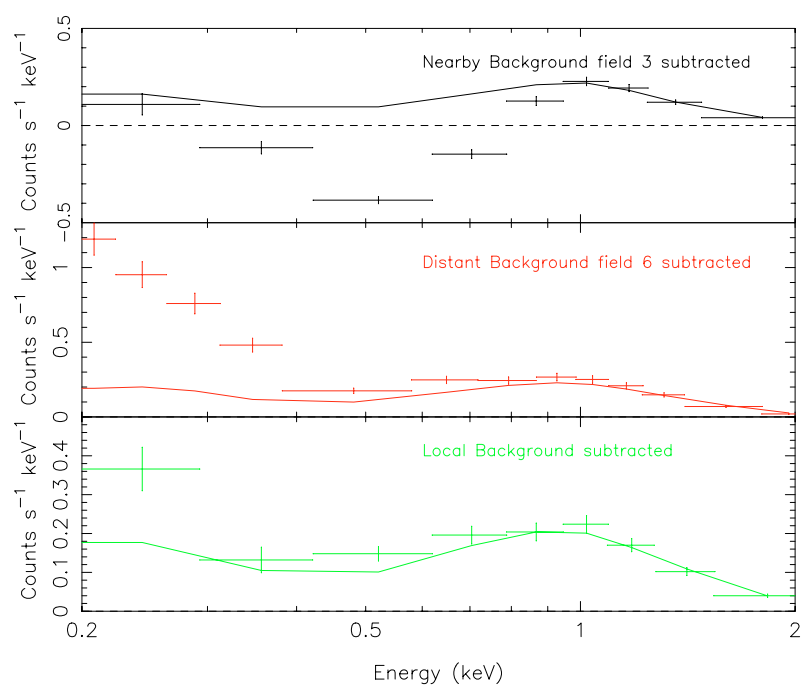

Fig. 9. PSPC spectrum of the $8-12$ arcmin annulus of A2052. The solid line is the best-fit model of the hot ICM emission, obtained by a fit to the 1-2 keV spectrum. After the fit, spectra were rebinned so that each datapoint has $S / N=10$. The use of nearby background 3 yields a significant oversubtraction, while use of background field 6 result in a strong soft excess emission, similar to the XMM results of Kaastra et al. (2003a).

subtracted from the cluster spectra in order to assess the presence of cosmological soft excess. Since the North Polar Spur is a region of enhanced and non-uniform soft X-ray emission, the most accurate background in this case is the local background from the same cluster observation. In this case, soft excess fluxes are detected, although with low statistical significance (Figs. 5 and 9c). Use of other nearby background fields at $\leq 10$ degrees distance from the clusters often yields oversubtraction of soft X-rays at large radii ( $\eta \leq 0$, Figs. 6 and 9a), that can be explained as inhomogenieties of the NPS soft X-ray emission on scales of few degrees, as shown in Fig. 1.

(b) All of the enhanced NPS X-ray emission is of extragalactic origin, and as such contributing to the cosmological soft excess. In this case one needs to apply a distant background, which results in the presence of strong cluster soft excess in all three clusters (Figs. 7 and 9b). In their analysis of XMM-Newton data of MKW03s and A2052, Kaastra et al. (2003a,b) followed this approach. They used background fields at high Galactic latitude, not contiguous to the cluster fields, and found strong soft excess emission. The soft excess emission we detect with this background subtraction method is consistent with that of XMM-Newton (Figs. 15, 16, 18 and 19 of Kastra et al. 2003a). In this case, the statistical significance of the $R 2$ band excess is greater that $10 \sigma$ for each cluster (Table 4). The excess is indeed "soft", i.e., predominantly found in the $R 2$ band.

(c) Part of the NPS emission is of Galactic origin, and part is of extragalactic origin. This scenario, tentatively surmised by Kaastra et al. (2003a) and Kuntz et al. (2001), is intermediate between (a) and (b): it would and yield positive soft excess fluxes, although lower than in case (b) above.

\subsection{Soft excess emission and associated OVII emission lines in the Hercules supercluster}

The PSPC data analyzed in this paper reveal the complexity of the soft X-ray sky in the direction of the Hercules supercluster, and the difficulty of establishing the intrinsic soft X-ray fluxes from the 3 galaxy clusters, MKW03s, A2052 and A2063. We conclude that:

(1) The PSPC data provide supporting evidence that the three galaxy clusters in the Hercules concentration feature the soft excess phenomenon. The evidence lies in the fact that the most conservative background subtraction method (local background) leads to positive soft excess fluxes in two of the three galaxy clusters (Figs. 5 and 9c).

(2) The strong soft excess radiation measured by the XMMNewton observations of Kaastra et al. (2003a,b) is of cluster origin only if the enhancement of the NPS soft X-rays is extragalactic. In this case, the 3 clusters we analyzed in the Hercules concentration feature strong soft excess emission, and the PSPC excess we measure (Fig. 7) matches the XMMNewton results of Kaastra et al. (2003a).

(3) If the soft X-ray radiation from the NPS is of local origin, the OVII emission lines of Kaastra et al. (2003a) are not associated with the soft cluster component. Our PSPC data show that $R 4$ band fluxes in the neighborhood of the Hercules supercluster can be few times higher than in other directions, with a standard deviation of $\sigma_{R 4}=81 \%$ for the 11 regions within 20 degrees of the three clusters. This level of uncertainty is significantly larger than that allowed by Kaastra et al. (2003a), who used a background at high Galactic latitude and allowed for a $35 \%$ systematic uncertainty, and it would result in their OVII emission lines not constituting a significant detection (see Fig. 7 of Kaastra et al. 2003a). This conclusion is confirmed by the fact that Mittaz et al. (2005) use a local background in their analysis of the XMM-Newton data of MKW03s and A2052 and detect no OVII emission lines in these clusters.

In the following we assume that the correct background is either the local one or the distant one (specifically, we choose field 6 as representative of the scenario (b)) and constrain the physical state of the soft excess emitter under these two assumptions. It is possible that the "true" background is somewhat intermediate between these two cases (scenario (c)): in 
this case, our mass estimates below will be interpreted as lower and upper limits, respectively.

\section{Interpretation}

\subsection{Nature and physical conditions of the soft excess emitter}

We interpret the excess emission as radiation from a warm plasma, which may reside inside the cluster (i.e., mixed with the hot ICM) or in filamentary structures that connect the clusters. The presence of a diffuse warm-hot intergalactic medium (WHIM: $T \sim 10^{5}-10^{7} \mathrm{~K}$ ) is predicted by several hydrodynamical simulations (e.g., Cen et al. 2001; Davé et al. 2001). Nevalainen et al. (2003), Mittaz et al. (2004) and Bonamente et al. (2005) discuss the WHIM interpretation of the soft excess, finding that the detected soft excess would imply WHIM filaments more massive and more extended than prediceted by numerical simulations. The detection of several absorption line systems in the optical, UV and X-ray spectra of quasars and AGNs is commonly interpreted with the detection of the WHIM (e.g., Tripp et al. 2000; Oegerle et al. 2000; Tripp \& Savage 2000; Savage et al. 2002; Sembach et al. 2001; Shull et al. 2003). The filaments may concentrate towards clusters (Davé et al. 2001), where their emission level may become detectable at EUV and soft X-ray energies and cause the soft excess phenomenon in galaxy clusters.

We therefore use a 2-temperature optically-thin plasma model with Galactic absorption for the PSPC spectra (see Sect. 5.1), employing both the local background and background from field 6 . We fit the $0.2-2 \mathrm{keV}$ PSPC spectra by fixing the temperature and abundances of the hot phase at the bestfit values of Table 3, and assuming an abundance of $A=0.1$ for the warm-hot phase, in accord with the results of Kaastra et al. (2003a), Nevalainen et al. (2003) and Bonamente et al. (2003). In some regions of limited $\mathrm{S} / \mathrm{N}$ we fix the temperature of the warm gas at $k T_{\text {warm }}=0.2 \mathrm{keV}$ (see references above). Results are shown in Table 5. The warm phase has temperature $k T \leq 0.3 \mathrm{keV}$, one order of magnitude cooler than the hot ICM phase (Table 3 ).

\subsection{Mass estimates for the warm gas}

Following the method developed in Bonamente et al. (2003) we derive mass estimates for the warm emitter. If the soft excess emission originates from a warm phase of the ICM, the ratio between the emission integral of the hot ICM and the emission integral of the warm gas (see Table 5) measures the relative mass of the two phases. The emission integral is defined as

$I=\int n^{2} \mathrm{~d} V$

where $n$ is the gas density and $\mathrm{d} V$ is the volume of the emitting region; $I$ is measured by fitting the $\mathrm{X}$-ray spectrum (see Table 5). We assume that the hot and warm phases have a volume filling factor of 1.0 and that each annulus corresponds to a spherical shell ${ }^{2}$. The mass ratio $\frac{M_{\text {warm }}}{M_{\text {hot }}}$ between the two phases (Table 6) is given by

$\frac{M_{\text {warm }}}{M_{\text {hot }}}=\frac{\int n_{\text {warm }} \mathrm{d} V}{\int n_{\text {hot }} \mathrm{d} V}=\frac{\int \mathrm{d} I_{\text {warm }} / n_{\text {warm }}}{\int \mathrm{d} I_{\text {hot }} / n_{\text {hot }}}$.

The density of the warm phase is lower than that of the hot ICM at small radii, and becomes comparable or larger at large radii. In this simple model the two phases share the same volumes, and they are not in pressure equilibrium. This implies a dynamical scenario in which the low-pressure warm gas is infalling into the cluster potential well.

The warm gas may however be distributed in extended low-density filaments (the WHIM), in accordance to the recent large-scale simulations. Following this scenario, we cannot measure at the same time the size and the density of the filaments (Bonamente et al. 2005). We assume a fiducial density of $n_{\text {fil }}=10^{-4} \mathrm{~cm}^{-3}$, corresponding to an overdensity of $\delta \sim 300$, and use

$\frac{M_{\mathrm{fil}}}{M_{\mathrm{hot}}}=\frac{\int n_{\mathrm{fil}} \mathrm{d} V}{\int n_{\mathrm{hot}} \mathrm{d} V}=\frac{\int \mathrm{d} I_{\text {warm }} / n_{\mathrm{fil}}}{\int \mathrm{d} I_{\text {hot }} / n_{\mathrm{hot}}}$

to measure that filament to hot ICM mass ratio (Table 6). Using this assumption on the density one can estimate the length of the filaments along the line of sight for each annulus:

$L_{\mathrm{fil}}=\frac{I_{\mathrm{warm}}}{n_{\mathrm{fil}}^{2} \cdot A}$

where $A$ is the area of the annulus. Using the distant background-subtracted $12-18^{\prime}$ of A2052 as a representative region, one finds that a filament of length $L_{\mathrm{fil}}=27-39 \mathrm{Mpc}$ is required to explain the detected emission. If filaments are less dense, the size would further increase according to $L_{\text {fil }} \propto n_{\text {fil }}^{2}$ (Eq. (4)) and the corresponding mass estimates would increase according to $M_{\text {fil }} \propto n_{\text {fil }}$. Such filaments would challeng our understanding of large-scale structures and are similar in size to those needed to explain the soft excess emission detected in the XMM data of Sèrsic 159-03 (Bonamente et al. 2005).

In Table 6 we also show the hot ICM and total gravitational masses for the three clusters, based on the Einstein IPC data of White et al. (1997). They indicate that the hot ICM accounts for about $10 \%$ of the total mass, out to the radii of their measurements. The warm gas may be several times as massive as the hot gas (Table 6), and so explain the missing baryons in the (super)cluster region.

\subsection{Non-thermal scenario}

It is in principle possible to explain the soft excess emission in the Hercules supercluster as non-thermal radiation, i.e., using a power-law model instead of a second thermal model for the $0.2-2 \mathrm{keV}$ PSPC spectra. The fit of our PSPC spectra with such a non-thermal model are usually statistically acceptable (not shown in Table 5). The major challenge of this scenario

\footnotetext{
2 A deprojection method would be more accurate to measure plasma densities. The method is however sufficient for the purpose of deriving $\frac{M_{\text {warm }}}{M_{\text {hot }}}$, which is the aim of this analysis.
} 
Table 5. Soft excess emitter.

\begin{tabular}{|c|c|c|c|c|c|c|}
\hline $\begin{array}{r}\text { Region } \\
(\text { arcmin })\end{array}$ & $\begin{array}{c}k T_{\text {warm }}^{a} \\
(\mathrm{keV})\end{array}$ & $\begin{array}{c}n_{\text {warm }} \\
\left(10^{-3} \mathrm{~cm}^{-3}\right)\end{array}$ & $I_{\text {warm }}$ & $\chi^{2}$ (d.o.f.) & $\begin{array}{c}n_{\mathrm{hot}} \\
\left(10^{-3} \mathrm{~cm}^{-3}\right)\end{array}$ & $I_{\text {hot }}^{b}$ \\
\hline \multicolumn{7}{|c|}{ MKw03s } \\
\hline $0-2 \mathrm{~L}$ & - & - & - & - & 7.50 & $1.5 \times 10^{-2}$ \\
\hline $0-26$ & - & - & - & - & 7.50 & $1.5 \times 10^{-2}$ \\
\hline $2-4 \mathrm{~L}$ & $\leq 0.26$ & $\leq 1.04$ & $\leq 2.0 \times 10^{-3}$ & $122.5(113)$ & 2.16 & $8.7 \times 10^{-3}$ \\
\hline $2-46$ & $\leq 0.15$ & $0.77-1.50$ & $1.1-4.2 \times 10^{-3}$ & $116.3(112)$ & 2.27 & $8.8 \times 10^{-3}$ \\
\hline $4-8 \mathrm{~L}$ & - & - & - & - & 0.69 & $7.2 \times 10^{-3}$ \\
\hline $4-86$ & $\leq 0.10$ & $0.68-0.86$ & $7.0-11.0 \times 10^{-3}$ & $123.7(111)$ & 0.71 & $7.6 \times 10^{-3}$ \\
\hline $8-12 \mathrm{~L}$ & - & - & - & - & 0.29 & $3.4 \times 10^{-3}$ \\
\hline $8-126$ & $\leq 0.13$ & $0.40-0.59$ & $6.5-14.0 \times 10^{-3}$ & $85.0(87)$ & 0.31 & $4.0 \times 10^{-3}$ \\
\hline $12-18 \mathrm{~L}$ & - & - & - & - & 0.10 & $1.5 \times 10^{-3}$ \\
\hline $12-186$ & $\leq 0.09$ & $0.46-0.53$ & $2.9-3.8 \times 10^{-2}$ & $129.4(106)$ & 0.14 & $2.7 \times 10^{-3}$ \\
\hline \multicolumn{7}{|c|}{ A2052 } \\
\hline $0-2 \mathrm{~L}$ & 0.2 & $\leq 1.9$ & $\leq 0.75 \times 10^{-3}$ & $161.9(135)$ & 9.22 & $1.8 \times 10^{-2}$ \\
\hline $0-26$ & 0.2 & $\leq 1.9$ & $\leq 0.75 \times 10^{-3}$ & $165.2(135)$ & 9.22 & $1.8 \times 10^{-2}$ \\
\hline $2-4 \mathrm{~L}$ & - & - & - & - & 2.84 & $1.2 \times 10^{-2}$ \\
\hline $2-46$ & $\leq 0.14$ & $0.63-1.70$ & $0.59-4.3 \times 10^{-3}$ & 119.6(104) & 2.84 & $1.2 \times 10^{-2}$ \\
\hline $4-8 \mathrm{~L}$ & $\leq 0.19$ & $0.26-0.73$ & $0.8-6.3 \times 10^{-3}$ & $104.3(115)$ & 0.96 & $1.1 \times 10^{-2}$ \\
\hline $4-86$ & $\leq 0.12$ & $0.87-1.25$ & $8.9-18.7 \times 10^{-3}$ & $105.9(116)$ & 0.92 & $1.0 \times 10^{-2}$ \\
\hline $8-12 \mathrm{~L}$ & $\leq 0.24$ & $0.18-0.49$ & $1.0-7.6 \times 10^{-3}$ & $85.7(76)$ & 0.37 & $4.5 \times 10^{-3}$ \\
\hline $8-126$ & $\leq 0.11$ & $0.68-0.95$ & $1.5-2.9 \times 10^{-2}$ & $88.6(76)$ & 0.35 & $4.0 \times 10^{-3}$ \\
\hline $12-18 \mathrm{~L}$ & - & - & - & - & 0.18 & $3.6 \times 10^{-3}$ \\
\hline $12-186$ & $\leq 0.10$ & $0.56-0.67$ & $3.4-4.9 \times 10^{-2}$ & $105.4(96)$ & 0.21 & $4.6 \times 10^{-3}$ \\
\hline \multicolumn{7}{|c|}{ A2063 } \\
\hline $0-2 \mathrm{~L}$ & $\leq 0.17$ & $1.2-3.8$ & $0.31-3.0 \times 10^{-3}$ & $139.0(121)$ & 6.59 & $9.2 \times 10^{-3}$ \\
\hline $0-26$ & $\leq 0.14$ & $2.17-4.35$ & $1.0-4.0 \times 10^{-3}$ & $139.4(121)$ & 6.59 & $9.2 \times 10^{-3}$ \\
\hline $2-4 \mathrm{~L}$ & $\leq 0.12$ & $0.61-1.54$ & $0.55-3.5 \times 10^{-3}$ & $137.3(126)$ & 2.60 & $1.0 \times 10^{-2}$ \\
\hline $2-46$ & $\leq 0.10$ & $1.54-2.14$ & $3.5-6.8 \times 10^{-3}$ & $136.3(126)$ & 2.60 & $1.0 \times 10^{-2}$ \\
\hline $4-8 \mathrm{~L}$ & $\leq 0.16$ & $0.46-0.84$ & $2.5-8.4 \times 10^{-3}$ & $135.1(147)$ & 1.00 & $1.2 \times 10^{-2}$ \\
\hline $4-86$ & $\leq 0.13$ & $0.92-1.20$ & $1.0-1.7 \times 10^{-2}$ & $141.2(147)$ & 1.00 & $1.2 \times 10^{-2}$ \\
\hline $8-12 \mathrm{~L}$ & $\leq 0.27$ & $0.14-0.42$ & $0.62-5.6 \times 10^{-3}$ & $93.4(121)$ & 0.41 & $5.3 \times 10^{-3}$ \\
\hline $8-126$ & $\leq 0.12$ & $0.66-0.86$ & $1.4-2.4 \times 10^{-2}$ & $98.0(120)$ & 0.42 & $5.8 \times 10^{-3}$ \\
\hline $12-18 \mathrm{~L}$ & 0.2 & $\leq 0.13$ & $\leq 1.8 \times 10^{-3}$ & $124.5(125)$ & 0.19 & $3.8 \times 10^{-3}$ \\
\hline $12-186$ & $\leq 0.10$ & $0.58-0.70$ & $3.6-5.4 \times 10^{-2}$ & $166.2(124)$ & 0.21 & $5.0 \times 10^{-3}$ \\
\hline
\end{tabular}

${ }^{a}$ The XSPEC fitting program operates only at temperatures $\geq 81 \mathrm{eV}$.

${ }^{b} I$ is the best-fit emission integral in the units of $10^{14}\left[4 \pi((1+z) D)^{2}\right] \mathrm{cm}^{-3}$, where $D$ is the distance to the source (in $\mathrm{cm}$ ) and $z$ is the redshift. See the XSPEC manual at http://heasarc.gsfc nasa.gov/docs/xanadu/xspec/manual/manual.html. $90 \%$ confidence intervals are shown.

L corresponds to local background subtraction, 6 to background subtraction using field 6 .

is explaining the presence of non-thermal, relativistic electrons located at large distances from the cluster centers (see, e.g., Bonamente et al. 2001c, 2002, 2003; Sarazin \& Lieu 1998 for discussion of the non-thermal model). We therefore do not further consider the non-thermal interpretation of the soft excess emission from the Hercules superclusters in this paper.

\subsection{Comparison with XMM results}

Kaastra et al. (2003b) find a typical density of $\sim 10^{-4} \mathrm{~cm}^{-3}$ for the soft excess component in MKW03s and A2052. Their estimates assume a constant-density warm gas distributed over a sphere of $\sim 1$ degree radius, and the emission from each annulus originates from a volume $V_{f}=2 \pi r \mathrm{~d} r \times(2 z)$, where $z^{2}+r^{2}=R^{2}$, and $R$ is a fiducial extent of the soft excess ( $\sim 1$ degree). Our density estimates of Table 5 assume that the emission originates from within the cluster, i.e., from a volume $V=4 \pi r^{2} \mathrm{~d} r$. For, e.g., $r=10 \mathrm{arcmin}$ and $R=60 \mathrm{arcmin}$, the ratio of the two volumes is $V_{f} / V \sim 6$, and a 6 times smaller volume implies a density $\sqrt{6} \sim 2.5$ times higher to explain the same emission integral. This is why the estimates of Table 5 exceed those of Kaastra et al. (2003b) by a factor of few, when we employ the distant background.

\section{Conclusions}

PSPC observations of three clusters in the Hercules concentration, MKW03s, A2052 and A2063, and of 8 background fields 
Table 6. Mass estimates of the warm emitter.

\begin{tabular}{rcccc}
\hline \hline Cluster $^{a}$ & $\frac{M_{\text {warm }}}{M_{\text {hot }}}$ & $\frac{M_{\text {fil }}}{M_{\text {hot }}}$ & $M_{\text {hot }}^{b}$ & $M_{\text {tot }}^{b}$ \\
\hline MKW03s (L) & $\leq 0.10$ & $\leq 0.55$ & $27.9 \pm 2.5$ & $248^{c}$ \\
MKW03s (6) & $1.9-2.3$ & $8.9-13.8$ & & \\
A2052 (L) & $0.18-0.50$ & $0.36-2.97$ & $30.8 \pm 2.7$ & $220^{d}$ \\
A2052 (6) & $1.85-2.39$ & $11.5-20.0$ & & \\
A2063 (L) & $0.22-0.80$ & $0.79-4.41$ & $4.7 \pm 0.2$ & $63^{e}$ \\
A2063 (6) & $1.80-2.27$ & $11.9-19.5$ & & \\
\hline
\end{tabular}

a "L" indicates local background and 6 indicates background subtraction using the "distant" field 6. Errors are 90\% confidence, based on the $90 \%$ confidence intervals of the emission integrals of Table 5.

${ }^{b}$ Masses are in units of $10^{12} M_{\odot}$. Reference: White et al. (1997).

${ }^{c}$ Masses are calculated out to a radius of $0.76 \mathrm{Mpc}$.

${ }^{d}$ Masses are calculated out to a radius of $0.89 \mathrm{Mpc}$.

${ }^{e}$ Masses are calculated out to a radius of $0.28 \mathrm{Mpc}$.

in their vicinity were used to study the presence of soft excess emission. We showed that the Hercules supercluster lies in a region of the sky - the southern extension of the North Polar Spur - that has strong and inhomogenous soft X-ray emission.

The soft excess fluxes of MKW03s, A2052 and A2063 depend on the nature of the North Polar Spur. If the soft Xray emission from the NPS is of local origin, only A2052 and A2063 show evidence of cluster soft excess. In this case, the soft excess fluxes we measure with PSPC are lower than those reported in the XMM study of Kaastra et al. (2003a), and the OVII emission lines they detected towards MMKW03s and A2052 are not associated with the clusters. If part or all of the NPS emission is of extragalactic origin, the 3 clusters feature strong soft excess emission, in accord with the XMM results.

The soft excess emission is interpreted with the presence of warm gas that can be either intermixed with the hot gas, or located in filamentary structures around the clusters. The PSPC data show that the warm gas feature a temperature of $k T \leq$ $0.3 \mathrm{keV}$. If the warm gas is intra-cluster, it is approximately as massive as the hot ICM, and significantly more massive if located in filamentary structures around the clusters.

\section{References}

Blanton, E. L., Sarazin, C. L., \& McNamara, B. R. 2003, ApJ, 585, 227

Bonamente, M., Lieu, R., \& Mittaz, J. P. D. 2001a, ApJ, 547, 7

Bonamente, M., Lieu, R., \& Mittaz, J. P. D. 2001b, ApJ, 561, 67

Bonamente, M., Lieu, R., Nevalainen, J., \& Kaastra, J. S. 2001c, ApJ, 552,7

Bonamente, M., Lieu, R., Joy, M. K., \& Nevalainen, J. H. 2002, ApJ, 576,688

Bonamente, M., Joy, M. K., \& Lieu, R. 2003, ApJ, 585, 722
Brinkmann, W., Grupe, D., Branduardi-Raymont, G., \& Ferrero, E. 2003, A\&A, 398, 81

Cen, R., \& Ostriker, J. P. 1999, ApJ, 514, L1

Cen, R., Tripp, T. M., Ostriker, J. P., \& Jenkins, E. B. 2001, ApJ, 559, L5

Davé, R., Cen, R., Ostriker, J. P., et al. 2001, ApJ, 552, 473

De Grandi, S., \& Molendi, S. 2002, ApJ, 567, 163

Dickey, J. M., \& Lockman, F. J. 1990, ARA\&A, 28, 215

Finoguenov, A., Arnaud, M., \& David, L. P. 2001, ApJ, 555, 191

Iwan, D. 1980, ApJ, 239, 316

Kaastra, J. S., Lieu, R., Bleeker, J. A. M., Mewe, R., \& Colafrancesco, S. 2002, ApJ, 574, L1

Kaastra, J. S., Lieu, R., Tamura, T., Paerels, F. B. S., \& den Herder, J. W. 2003a, A\&A, 397, 445

Kaastra, J. S., Lieu, R., Tamura, T., Paerels, F. B. S., \& den Herder, J. W. 2003b, in Soft X-ray emission from cluster of galaxies and related phenomena, ed. R. Lieu (Kluwer), in press [arXiv: astro-ph/0305424]

Kaastra, J. S., Tamura, T., Peterson, J. R., et al. 2004, A\&A, 413, 415

Kuntz, K. D., Snowden, S. L., \& Mushotzky, R. F. 2001, ApJ, 548, L119

Lieu, R., Mittaz, J. P. D., Bowyer, S., et al. 1996a, ApJ, 458, L5

Lieu, R., Mittaz, J. P. D., Bowyer, S., et al. 1996b, Science, 274, 1335

Lieu, R., Bonamente, M., \& Mittaz 1999, ApJ, 517, L91

Mittaz, J. P. D., Lieu, R., \& Lockman, F. J. 1998, ApJ, 498, L17

Mittaz, J. P. D., Lieu, R., \& Bonamente 2005, A\&A, submitted

Nicastro, F., Zezas, A., Elvis, M., et al. 2003, Nature, 421, 719

Nevalainen, J. H., Lieu, R., Bonamente, M., \& Lumb, D. 2003, ApJ, 514,716

Oegerle, W. R., et al. 2002, ApJ, 538, L23

Page, K. L., Schartel, N., Turner, M. J. L., \& O’Brien, P. T. 2004, MNRAS, 352, 523

Peterson, J. R., Kahn, S. M., Paerels, F. B. S., et al. 2003, ApJ, 590, 207

Plucinsky, P. P, Snowden, S. L., Briel, U. G., Hasinger, G., \& Pfefferman, E. 1993, ApJ, 418, 519

Sarazin, C. L., \& Lieu, R. 1998, ApJ, 494, L177

Savage, B. D., Sembach, K. R., Tripp, T. M., \& Richter, P. 2002, ApJ, 564,631

Sembach, K. R., Howk, J. C., Savage, B. D., Shull, J. M., \& Oegerle, W. R. 2001, ApJ, 561, 573

Snowden, S. L., McCammon, D., Burrows, D. N., \& Mendenhall, J. A. 1994, ApJ, 424, 714

Snowden, S. L., Freyberg, M. J., Plucinsky, P. P., et al. 1995, ApJS, 454,643

Snowden, S. L., Egger, R., Freyberg, M. J., et al. 1997, ApJ, 485, 125

Snowden, S. L., Egger, R., Finkbeiner, D. P., Freyberg, M. J., \& Plucinsky, P. P. 1998, ApJ, 493, 715

Snowden, S. L., Freyberg, M. J., Kuntsz, K. D., \& Sanders, W. T. 2000, ApJ, 128, 171

Shull, J. M., Tumlison, J., \& Giroux, M. L. 2003, ApJ, 594, L107

Tamura, T., Kaastra, J. S., den Herder, J. W. A., Bleeker, J. A. M., \& Peterson, J. R. 2003, A\&A, submitted

Tripp, T. M., Savage, B. D., \& Jenkins, E. B. 2000, ApJ, 534, 1

Tripp, T. M., Giroux, M. L., Stocke, J. T., Tumlison, J., \& Oegerle, W. R. 2001, ApJ, 563, 724

Tripp, T. M., \& Savage, B. D. 2000, ApJ, 542, 42

White, D. A., Jones, C., \& Forman, W. 1997, MNRAS, 292, 419 